\title{
SURFACES OF HARD-SPHERE SYSTEMS
}

\author{
DIETRICH STOYAN区 \\ Institut für Stochastik, Technische Universität Bergakademie Freiberg, D-09596 Freiberg, Germany \\ e-mail: stoyan@math.tu-freiberg.de \\ (Received March 4, 2014; revised June 17, 2014; accepted July 9, 2014)
}

\begin{abstract}
In various situations surfaces appear that are formed by systems of hard spheres. Examples are porous layers as surfaces of sand heaps and biofilms or fracture surfaces of concrete. The present paper uses a model where a statistically homogeneous system of hard spheres with random radii is intersected by a plane and the surface is formed by the spheres with centers close to this plane. Formulae are derived for various characteristics of such surfaces: for the porosity profile, i.e., the local porosity in dependence on the distance from the section plane and for the geometry of the sphere caps that are located above the section plane.

It turns out that these characteristics only depend on the first-order characteristics of the sphere system, its sphere density and the sphere radius distribution.

Comparison with empirically studied biofilms shows that the model is realistic.
\end{abstract}

Keywords: biofilm, fracture surface, hard spheres, porous layer, surface.

\section{INTRODUCTION}

Porous layers at surfaces of hard-sphere systems are observed in various contexts. An example is the liquid-gas interface as considered in Hansen and McDonald (1986), p. 136, where the liquid is modeled by a system of hard spheres. Another example is the interface between a fluid and a porous substrate which is modeled by a system of hard spheres, as in the case of seabed, biofilms and various technical applications, see Bondreau and Jorgensen (2001) and Lewandowski (2000). Finally, fracture surfaces in concrete are modeled in a similar way, where the hard spheres stand for the aggregate particles (Stroeven, 1982; 2000).

For such interfaces, in particular the porosity profile, i.e., the local porosity in direction of the normal to the surface plane as a function of the distance from the plane is of interest. The porosity is decreasing in the direction gas to liquid or substrate to fluid. Thus a gradient structure as in Hahn and Stoyan (1998) and Hahn and Stoyan (1998) is given.

The present paper considers porous layers that are generated by removing spheres from an originally spatially homogeneous (or stationary) system of hard spheres on one side of a plane. It gives formulae for the porosity profile and for sphere-cap characteristics in the general case of non-identical spheres.

It turns out that these characteristics only depend on the first-order characteristics of the sphere system: its sphere density and the sphere-radius distribution.

\section{HARD SPHERE SYSTEMS AND THREE BOUNDARY STRUCTURES}

The model of the sphere systems is the same as in Mecke and Stoyan (1980) and Chiu et al. (2013), p. 428 , the probably most general stochastic model for a stationary (= statistically homogeneous) system of spheres. There is a stationary marked point process $\Psi$ in $\mathbb{R}^{3}$,

$$
\Psi=\left\{\left[x_{n}, y_{n}, z_{n} ; \rho_{n}\right]\right\},
$$

where $\left(x_{n}, y_{n}, z_{n}\right)$ is the center of the $n$th sphere and the positive mark $\rho_{n}$ its radius. The intensity of $\Psi$ is $\lambda$, which represents the sphere center density or the mean number of sphere centers per volume unit. Finally, the mark distribution function, i.e., the radius distribution function, is denoted by $F(r)$ : the probability that a randomly chosen sphere has a radius smaller than $r$ is $F(r)$. The volume fraction of the sphere system is denoted by $p$.

In a hard sphere system the spheres do not intersect, i.e., it is

$B\left(\left(x_{n}, y_{n}, z_{n}\right), \rho_{n}\right) \cap B\left(\left(x_{m}, y_{m}, z_{m}\right), \rho_{m}\right)=\emptyset \quad$ for $m \neq n$,

where $B((x, y, z), \rho)$ is the sphere with center $(x, y, z)$ and radius $\rho$. The sphere points with maximum $z$ coordinate are called north poles.

Fig. 1 shows a simulated system of hard spheres in a box. Its irregular surfaces are closely related to the geometrical structures discussed in the following. 


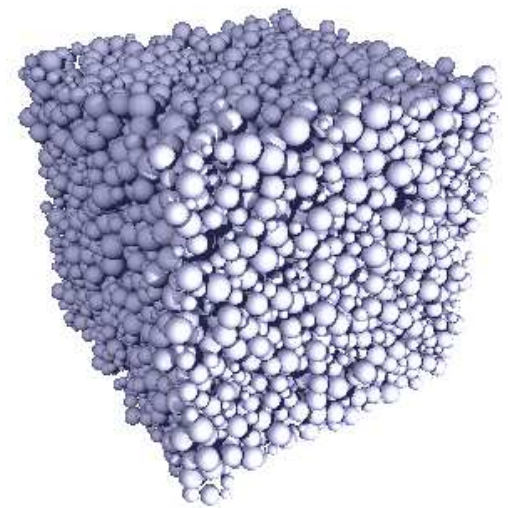

Fig. 1. Computer-simulated system of hard spheres in a box with periodic boundary conditions. The volume fraction is 0.6, the porosity 0.4. The structure was generated by means of the force-biased algorithm, see Bezrukov et al. (2002)

Three structures connected with the hard-sphere system are considered below which appear in the neighborhood of the $(x, y)$-plane $z=0$, which plays the role of the dividing plane. The first two are the boundaries of the following two sets:

- the up-cap plane set, the set-theoretic union of the half space $z \leq 0$ and all closed balls of $\Psi$ with centers $\left(x_{n}, y_{n}, z_{n}\right)$ below the $(x, y)$-plane $\left(z_{n} \leq 0\right)$ and radii $\rho_{n}$ with $\rho_{n}>\left|z_{n}\right|$. The space below the plane united with the protruding caps in Fig. 2 can be considered as a sample of an up-cap plane set;

- the up-down-cap set, i.e., up-cap plane set minus the union of all spheres of $\Psi$ with centers $\left(x_{n}, y_{n}, z_{n}\right)$ above the $(x, y)$-plane $\left(z_{n}>0\right)$ and radii $\rho_{n}$ with $\rho_{n}>z_{n}$. Fig. 2 shows a simulated sample of an up-down-cap set.

The upper (in the sense of $z$-axis) boundaries of these sets are called up-cap plane and up-down-cap plane.

The third structure is

- the free-boundary set, the system of all spheres with $z_{n}+\rho_{n} \leq 0$, see Fig. 3 .

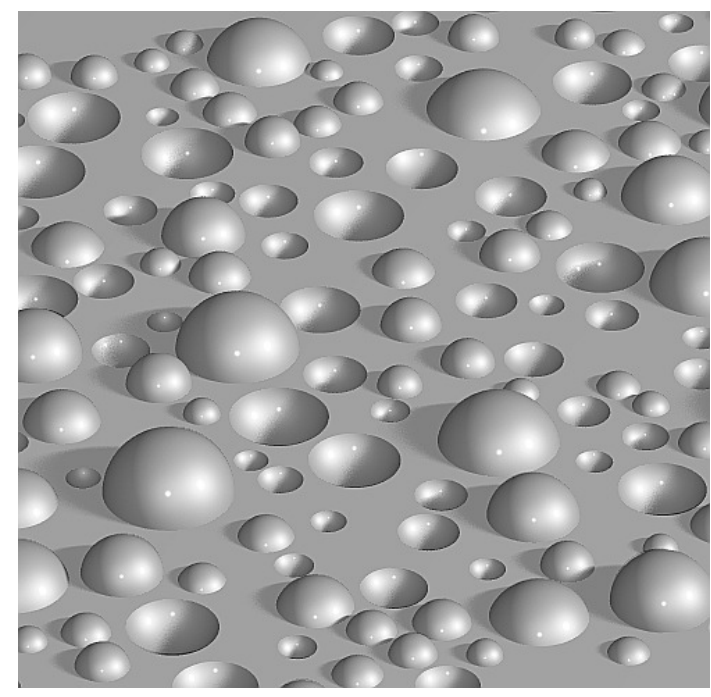

Fig. 2. Computer-simulated surface composed of interconnected portions of the dividing plane and protruding and indenting dome-like caps resulting from a hard sphere system, cf. Stroeven (2000) In the present paper it is called up-down-cap plane'. Courtesy of M. Stroeven.

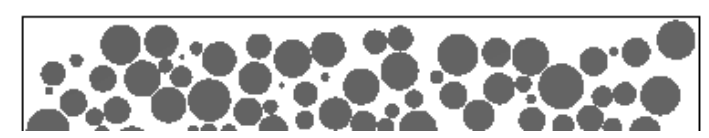

Fig. 3. Computer-simulated vertical section through a free-boundary set. It consists of all spheres below the $(x, y)$-plane. The upper horizontal line belongs to the $(x, y)$-plane. The volume fraction of the hard-sphere system is 0.6.

For the up-cap plane set two characteristics are determined:

- the intensity $\lambda_{m}(h)$ of the planar point process of north poles of protruding caps higher than $h$ (with $h>0)$ projected onto the $(x, y)$-plane;

- the density profile, i.e., the area fraction $a_{m}(h)$ of the planar subset $\Xi_{h}$ of the plane $\{z=h\}$ generated by intersection of the up-cap plane set with this plane. The porosity profile is given by $1-a_{m}(h)$.

The ratio $\lambda_{m}(h) / \lambda_{m}(0)$ yields the cap-height distribution function $H(h)$,

$$
H(h)=1-\lambda_{m}(h) / \lambda_{m}(0) \quad \text { for } h \geq 0 .
$$

The characteristics for the up-down-cap plane can be easily obtained from those of the up-cap plane.

For the free-boundary set the following characteristics are determined: 
- the density profile, i.e., the area fraction $a_{f}(h)$ of the planar subset of the plane $\{z=-h\}$ generated by intersection of the free-boundary set with this plane;

- the distribution function $F_{h}(r)$ of the radii of the spheres belonging to the free-boundary set with centers in the plane $z=-h$. (This is only of interest for random spheres since in the case of constant radius $R$ also the spheres with center at $z=-h$ have radius $R$.)

\section{RESULTS}

Theorem 1. The intensity $\lambda_{m}(h)$ for the up-cap plane is given by

$$
\lambda_{m}(h)=\lambda \int_{h}^{\infty}(r-h) \mathrm{d} F(r) \quad \text { for } h \geq 0,
$$

where $\lambda$ denotes the spheres center density and $F(r)$ the radius distribution function. The same formula holds in the planar case, for a marked point process $\left\{\left[x_{n}, y_{n} ; \rho_{n}\right]\right\}$ describing a system of discs and a up-cap line related to $\{y=0\}$.

Proof. Clearly, $\lambda_{m}(h)$ can be expressed in terms of the marked point process $\Psi$ as

$$
\lambda_{m}(h)=\mathbf{E}\left(\sum_{[x, y, z ; \rho] \in \Psi} \mathbf{1}_{[0,1]}(x) \mathbf{1}_{[0,1]}(y) \mathbf{1}_{[h-r, 0)}(z) \mathbf{1}_{[h, \infty)}(\rho)\right),
$$

since it equals the mean number of sphere centers with $(x, y)$ in the unit square $[0,1]^{2}$ and $z$-coordinate and radius $\rho$ satisfying

$$
z \leq 0 \text { and } h-z \leq r .
$$

The Campbell theorem for marked point processes (Chiu et al., 2013) yields

$$
\begin{aligned}
\lambda_{m}(h) & =\lambda \iint_{[h-r, 0]}(z) \mathrm{d} z \mathbf{1}_{[h, \infty)}(r) \mathrm{d} F(r) \\
& =\lambda \int_{h}^{\infty}(r-h) \mathrm{d} F(r) .
\end{aligned}
$$

In the planar case all goes analogously, in the expectation in Eq. 3 only one $\mathbf{1}_{[0,1]}$ term appears.

Clearly, Eq. 2 holds also without the hard sphere condition (Eq. 1). In the particular case of constant radii $R$ it is

$$
\lambda_{m}(h)= \begin{cases}\lambda(R-h) & \text { for } 0 \leq h \leq R \\ 0 & \text { otherwise. }\end{cases}
$$

Theorem 2. The area fractions for the up-cap plane and the free-boundary set are

$$
\begin{aligned}
a_{m}(h)= & \frac{\pi}{3} \lambda\left(\int_{0}^{h / 2} 4 r^{3} \mathrm{~d} F(r)+\right. \\
& \left.\int_{h / 2}^{\infty}(r-h)^{2}(2 r+h) \mathrm{d} F(r)\right) \quad \text { for } h \geq 0,
\end{aligned}
$$

and

$$
a_{f}(h)=\frac{\pi}{3} \lambda \int_{0}^{\infty} h^{2}(3 r-h) \mathrm{d} F(r) \quad \text { for } h \geq 0 .
$$

Proof of Eq. 5. Determining $a_{m}(h)$ is equivalent to determine the mean number of spheres hitting the point $(0,0, h)$, In fact, hitting happens if $\|(x, y, z)-$ $(0,0, h) \| \leq \rho$. Since the spheres are hard, only one sphere can hit a given point and the mean number is thus equal to the probability of hitting the point $(0,0, h)$ by one of the spheres. This probability is equal to the area fraction of the stationary random closed set $\Xi_{h}$ (the horizontal section set) because of equation (6.34) in Chiu et al. (2013), which says, applied to the case considered here, that "area fraction $a_{m}(h)=\mathbf{P}(o \in$ $\left.\Xi_{h}\right)$ ", where $o$ is the origin of the plane. Consequently,

$a_{m}(h)=\mathbf{E}\left(\sum_{[x, y, z ; \rho] \in \Psi} \mathbf{1}(\|(x, y, z)-(0,0, h)\| \leq \rho) \mathbf{1}_{(-\infty, 0]}(z)\right)$,

where $\|\cdot\|$ is the Euclidean norm. The Campbell theorem yields

$$
\begin{aligned}
& a_{m}(h)= \\
& =\lambda \int_{0}^{\infty} \int_{-\infty}^{0} \int_{-\infty}^{\infty} \int_{-\infty}^{\infty} \mathbf{1}(\|(x, y, z)-(0,0, h)\| \leq r) \mathrm{d} x \mathrm{~d} y \mathrm{~d} z \mathrm{~d} F(r) \\
& =\lambda \int_{h}^{\infty} V(r, h) \mathrm{d} F(r),
\end{aligned}
$$

where $V(r, h)$ is the volume of the cap of height $r-h$ (with $r \geq h$ ) of a sphere of radius $r$. Well-known formulas for sphere cap volume yield

$$
V(r, h)=\frac{\pi}{3}(r-h)^{2}(2 r+h) .
$$

The proof of Eq. 6 is analogous. Now the volume $V(r, h)$ is replaced by the volume of a sphere of radius $r$ minus a cap of height $2 r-h$. 
In the particular case of constant radii $R$ it is

$$
a_{m}(h)= \begin{cases}\frac{\pi}{3} \lambda(R-h)^{2}(2 R+h) & \text { for } 0 \leq h \leq R \\ 0 & \text { otherwise }\end{cases}
$$

and

$$
a_{f}(h)= \begin{cases}\frac{\pi}{3} \lambda h^{2}(3 R-h) & \text { for } 0 \leq h \leq 2 R \\ p & \text { otherwise }\end{cases}
$$

Fig. 4 shows $1-a_{f}(h)$ for $R=1$ and $\lambda=0.143$. For this intensity $\lambda$ the porosity of the hard-sphere system is 0.6 .

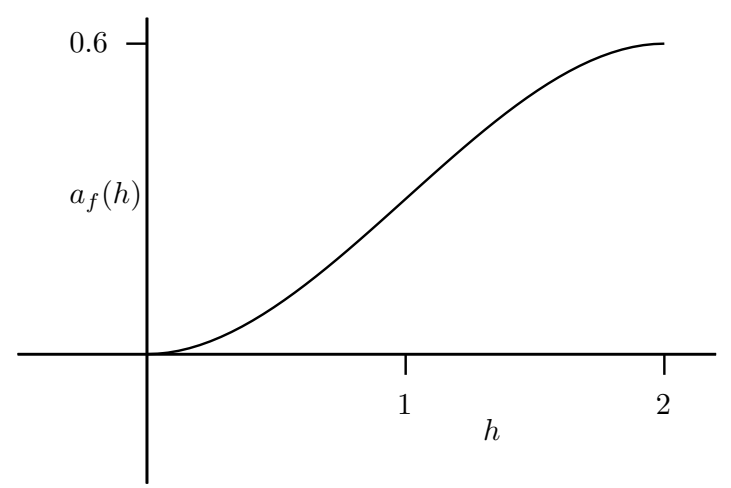

Fig. 4. Density profile $a_{f}(h)$ for the free boundary of $a$ hard sphere system with constant spheres of radius $R=1$ and porosity 0.4 .

Eq. 8 is derived in Khalili et al. (2014) without explicit use of point-process methods.

\section{DISCUSSION}

Fig. 4 resembles Fig. $5.6 \mathrm{a}$ in Hansen and McDonald (1986) which shows the density profile at the liquid-gas interface of the Lennard-Jones fluid (with identical spheres of radius $R$ ) near the triple point. However, while in Fig. 4 for the transition from density 0 to density 0.6 only $2 R$ length units are needed, in its counterpart in Hansen and McDonald (1986) they are 8R. By the way, Hansen and McDonald (1986) also discuss density profiles of Lennard-Jones fluids against a hard wall, which is much more complicated than the case considered in this paper.

Khalili et al. (2014) carried out experiments with natural packings of glass beads of (approximately) equal diameter in a container and with sedimented sand grains. With advanced physical measurement methods (laser technique and computerized tomography) they determined the porosity profile (i.e., $\left.1-a_{f}(h)\right)$ and found a good agreement with Eq. 8 for the case of constant radii (Fig. 5.)

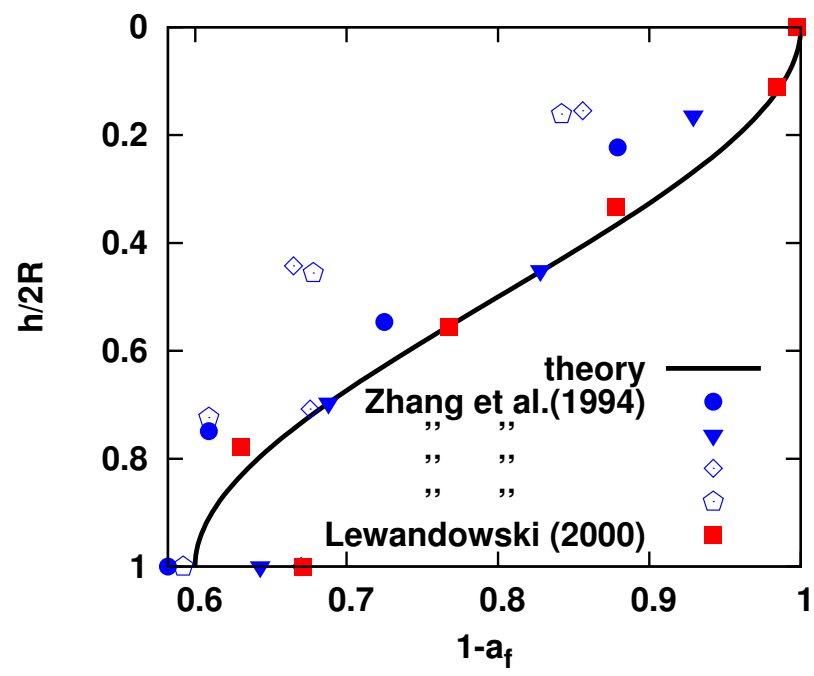

Fig. 5. Comparison of porosity profiles predicted from Eq. 8 with experimental porosity measurements within biofilms. The figure is a modification of Fig. 6 of Khalili et al. (2014). Courtesy of Arzhang Khalili.

\section{ACKNOWLEDGMENT}

The author thanks the two anonymous referees for their helpful and constructive remarks.

\section{REFERENCES}

Bezrukov A, Bargiel M, Stoyan D (2002) Statistical analysis of simulated random packings of spheres. Part Part Syst Char 19:111-8.

Bondreau BP, Jorgensen, BB (2001). The benthic boundary layer: transport processes and bio-geochemistry. Oxford: Oxford University Press.

Chiu SN, Stoyan D, Kendall WS, Mecke J (2013). Stochastic geometry and its applications, 3rd Ed. Chichester: Wiley.

Hahn U, Micheletti A, Pohlink R, Stoyan D, Wendrock H (1999). Stereological analysis and modeling of gradient structures. J Microsc 195:113-24.

Hahn U, Stoyan D (1998). Unbiased stereological estimation of the surface area of gradient surface processes. Adv Appl Prob 30:904-20.

Hansen JP, McDonald IR (1986). Theory of simple liquids. Academic Press, New York. 
Khalili A, Morad MR, Matyka M, Liu B, Malekmohammadi R, Weise J, Kuypers MMM (2013). Porosity variation below a fluid-porous interface. Chem Eng Sci 107:3116.

Lewandowski Z (2000). Notes on biofim porosity. Water Res 34:2620-4.

Mecke J, Stoyan D (1980). Stereological problems for spherical particles. Math Nachr 96:311-7.
Stroeven P (1982). Structural modelling of plain and fibre reinforced concrete. Composites 13:129-39.

Stroeven P (2000). A stereological approach to roughness of fracture surfaces and tortuosity of transport paths in concrete. Cement Concrete Comp 22:331-41.

Zhang TC, Bishop PL (1994). Density, porosity, and pore structure of biofilms. Water Res 28:2267-77. 